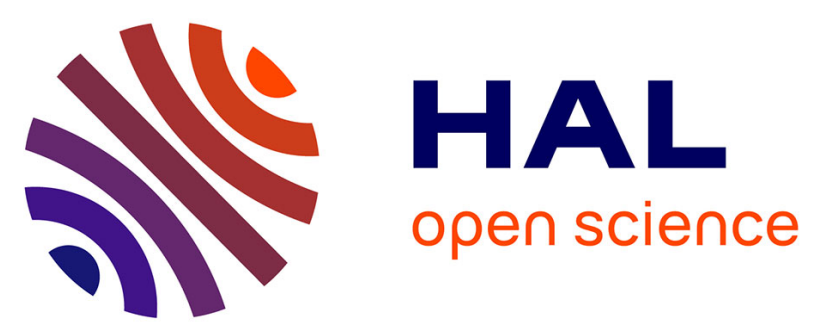

\title{
Design and Optimization of a Dextrous Robotic Finger: Incorporating a Sliding, Rotating, and Soft-Bending Mechanism While Maximizing Dexterity and Minimizing Dimensions
}

\author{
Amir Pagoli, Frédéric Chapelle, Juan Antonio Corrales Ramon, Youcef
}

\author{
Mezouar, Yuri Lapusta
}

\section{To cite this version:}

Amir Pagoli, Frédéric Chapelle, Juan Antonio Corrales Ramon, Youcef Mezouar, Yuri Lapusta. Design and Optimization of a Dextrous Robotic Finger: Incorporating a Sliding, Rotating, and Soft-Bending Mechanism While Maximizing Dexterity and Minimizing Dimensions. IEEE Robotics and Automation Magazine, 2020, 27 (4), pp.56-64. 10.1109/MRA.2020.3024283 . hal-03060366v2

\author{
HAL Id: hal-03060366 \\ https://hal.uca.fr/hal-03060366v2
}

Submitted on 5 Feb 2021

HAL is a multi-disciplinary open access archive for the deposit and dissemination of scientific research documents, whether they are published or not. The documents may come from teaching and research institutions in France or abroad, or from public or private research centers.
L'archive ouverte pluridisciplinaire HAL, est destinée au dépôt et à la diffusion de documents scientifiques de niveau recherche, publiés ou non, émanant des établissements d'enseignement et de recherche français ou étrangers, des laboratoires publics ou privés. 


\title{
Design and Optimization of a Robotic Finger with a Sliding, Rotating, and Soft-Bending Mechanism
}

\author{
A. Pagoli, F. Chapelle, J. Corrales, Y. Mezouar, Y. Lapusta
}

In this article, a new soft finger with a pneumatic-actuated movable joint is introduced, optimized and characterized in terms of degrees of freedom, workspace and fingertip force. The finger consists of one soft link as the body and the bending pneumatic joint as the actuator. Due to the additional translation and rotation movement capabilities of the joint carried out by two stepper motors, the finger can bend in any direction while having different lengths, thanks to a configurable bending point. This results in more dexterity of the finger dealing with a target inside its 3D workspace by increasing the number of configurations in which the finger can reach the target and exert force. The finite element method (FEM) and NSGA-II algorithm are applied to optimize the joint geometry to maximize the bending angle and minimize the joint dimensions. Furthermore, the variations of each design parameter and the consequent effects on the optimization objectives are analyzed. The optimal geometrical parameters are used to fabricate a prototype with silicone rubber. Tests on bending angle and tip force variability are conducted on the prototype to validate the numerical modeling. The experimental results show that the finger exerts force up to $650 \mathrm{mN}$ with a response time of less than three seconds. The stiffness of the finger can be changed by applying the pneumatic pressure in the hollow space inside the link. This consequently varies the amount of applied force at the tipping point of the finger up to two times.

\section{FEAs Actuators}

Introduced as a novel technology in recent years, soft robotics broadens new horizons in the field of robotics thanks to promising characteristics such as adaptability, lightweight, less assembly, and low cost [1]. The intrinsic deformable structure of soft robots encourages scientists to engage different technologies for their dynamization. One of the most widely used actuating technologies for soft robotics is Fluidic Elastomer Actuation (FEA), powered by a pressurized fluid (gas or liquid) [2]. Due to many advantages of FEAs including easy fabrication, producing high forces, large strokes, and lowcost elastomer materials [3], they have been used in numerous configurations for various purposes such as locomotion [4], manipulation [5], medical applications [6], and wearable devices [7]. These actuators can generate distributed forces which are proportional to the operating pressure of the fluid and the surface area on which the pressure is applied [8]. Even though there is a large diversity of applications for FEAs, many challenges remain in this field including stiffness control and shape configuration. Researches have increased the performance of these kinds of actuators by integrating them with other types of actuation methods that help FEAs in terms of shape control and variable stiffness. These lateral technologies are mainly based on using variable stiffness materials, including shape memory polymers (SMPs) [9], combinations of SMPs with thermoplastic polyurethane (TPU) [10], and low melting point alloys (LMPAs) [11]. The main drawbacks of SMPs are a high hysteresis and a low actuation speed differs from 5 to 60 seconds regarding to the size of the actuator [3]. Using LMPAs is another suggested method for changing bending point and shape configuration in FEAs. Applying an electric current to the alloy and heating, the structure phase changes locally from rigid to soft and thus, variable stiffness can be achieved [12]. Like SMPs, the transition time is the main issue in LMPAs. Depending on size and geometry, the melting time for LMPAs differs from 1 to 30 seconds, while cooling takes over 60 seconds [13].

We introduce in this article a novel type of soft finger based on bending point control and variable stiffness. The proposed finger is more flexible than previous solutions in terms of the attainable 3D space and applicable contact forces at the fingertip by changing the position of its joint, and thus, the bending point. The design consists of one elastomer tube as the soft link and one movable soft joint as the actuator. Applying the air pressure to the joint, the joint and the link will bend concurrently. Two stepper motors are responsible for moving the joint longitudinally along the link as well as rotating that around its axis. The joint can thus change the effective length of the finger and the bending direction. Unlike the previously proposed integrating methods with FEAs, based on SMPs or LMPAs, the position of the bending point is movable along the length of the link, which makes the finger more dexterous and reconfigurable.

Due to the nonlinear behavior of FEAs, their performance strongly depends on the geometry and dimensions of the actuator. Elsayed et al. [14] showed the effects of the position and shape configuration of the chamber on bending direction and angle value; they deployed a Finite Element Method (FEM) to study and optimize these design parameters. Decroly et al. [15] conducted an optimization study using a numerical model to miniaturize FEAs to be applicable in minimally invasive surgery. In our work, developing an optimization procedure is also essential for the achievement of our operating objectives: reconfigurability and variable stiffness. The NSGA-II algorithm is chosen as the optimization method due to its fast non-dominated sorting approach, fast crowded distance estimation procedure and simple crowded comparison operator [16]. We use these capabilities for maximizing the bending angle up to 90 degrees and simultaneously minimizing the length and diameter of the joint while dealing with a variety of design parameters. Moreover, we investigate the sensitivity of each design parameter to reduce the computational cost and thus increase the convergence speed of the design procedure.

In the following section, we discuss the conceptual design of the proposed finger, followed by the description of the optimization process. The design parameters are optimized to 
a)

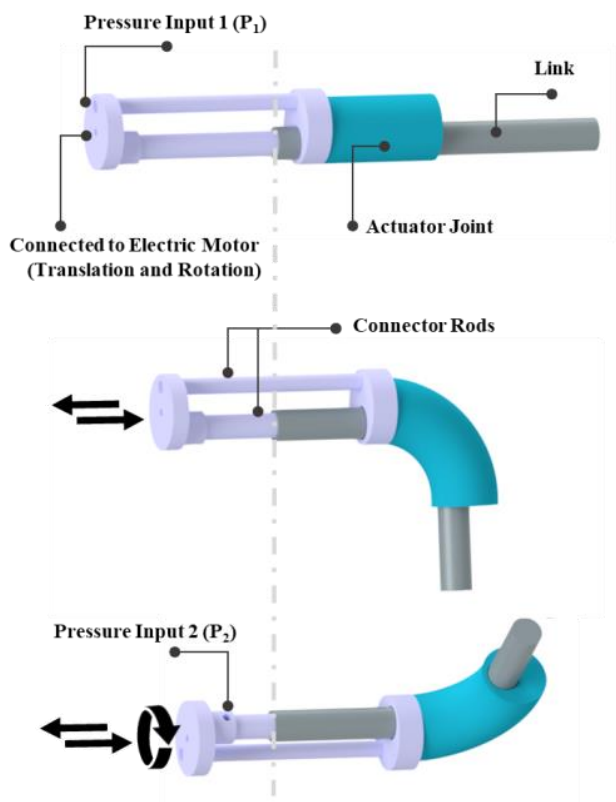

Figure 1. a) Schematic view of the proposed finger. b) sliding the joint along the link will change the bending point and the effective length of the finger. c) rotation of the joint along with its axis results in changing the bending direction in $3 \mathrm{D}$ space.

guarantee a correct bending operation and reduce the joint dimensions. Permissible stress and required pressure are also taken into account. Numerical validation and experimental results are presented in the results and discussions section. The workspace of the optimized finger is determined to evaluate its dexterity compared to a conventional finger. Experiments are then conducted to validate the reconfigurability function and to verify the possibility of obtaining different shape configurations with various exerted forces at the same target position. The article ends with conclusions and discussions over the future works.

\section{Operating Principles and Design}

The schematic of the proposed soft finger is illustrated in Figure 1. The finger is composed of a pneumatically actuated joint (blue cylinder) and a soft link (gray cylinder). A longitudinal channel is embedded inside the joint which inflates by supplying the air pressure $\left(P_{1}\right)$ and leads to the bending of the joint and consequently, the link (Figure 1a). The bending location can be longitudinally changed by sliding the joint along the link (Figure 1b). The joint can also rotate around its main axis while the link remains steady due to its fixed connection to the base. This causes the finger to bend in any direction in 3D space (Figure 1c).

In Figure 2a, a conventional FEA finger (on the right) and the proposed finger (on the left) are compared in terms of dexterity and the strategies to reach a particular point in the workspace. Due to the uniform structure of the conventional FEA fingers and their limited degree of freedom, it is not possible for them to attain each point in their workspace with various configurations. On the contrary, the design of the proposed finger suggests an array of possible configurations in which the finger can reach each point. This not only results in more flexibility of the finger in dealing with obstacles that a)

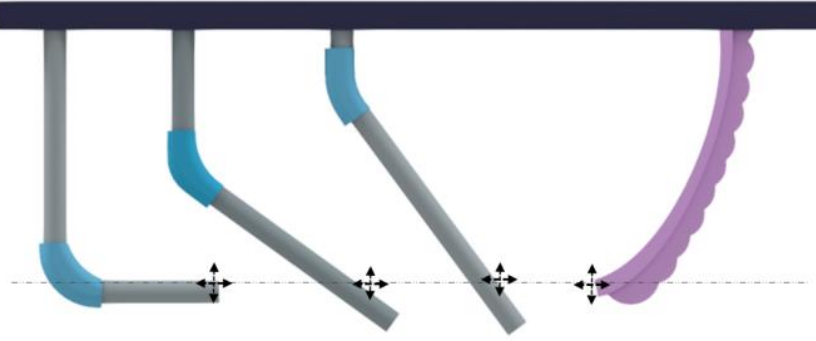

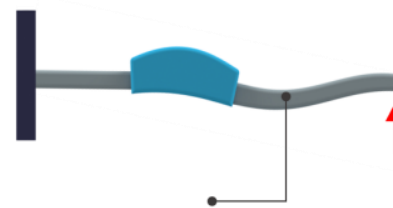

The stiffness of the finger can

b) be varied by applying pressure inside the soft link
The position of the joint and the stiffness of the finger, change the amount of exerted force at the tipping point.
Figure 2. Comparison between the configurations of the proposed finger (left) and a conventional FEA-based finger (right) a) reaching a particular point. b) exerting a different amount of force to a tipping point.

limit the workspace but also enhances the possible configurations in which the finger can exert a different amount of force to a particular point (Figure 2b). The workspaces of these two fingers will be compared in the results and discussion section. The mechanism used for changing the bending point of the finger mainly consists of two stepper motors connected to the joint (Figure 3). The first stepper motor is linked directly to the joint and is responsible for its rotational displacement. The connection includes two rigid rods; one for transmitting the rotational movement of the motor to the joint and the other with a tubular shape passing through the center of the link, for enhancing the stiffness of its region between the vertical support and the joint, which facilitates its deformation downstream and improves its controllability. As for the linear longitudinal movement of the joint, the assembly of the joint and the first motor are entirely displaced by the second stepper motor using a ball screw

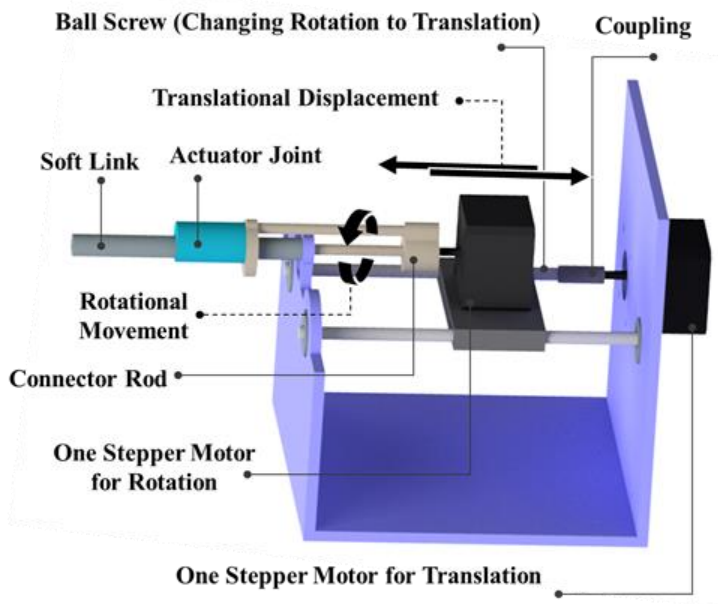

Figure 3. Assembly structure of the proposed finger and the motors. 
mechanism. We chose using a stepper motor solution for the movable joint due to its position accuracy and fast reactivity. Two air streams with different pressures are supplied to the finger; $P_{1}$ which deforms the joint and $P_{2}$ regulates the stiffness of the link. These two air streams are applied to the joint and the link via the two aforementioned rods between the joint and the stepper motor for rotation.

\section{Design Optimization}

\section{Finite Element Modeling}

In this section, the optimization procedure is described for a finger with the approximate dimensions of the human finger (diameter $=10 \mathrm{~mm}$ and length=150 $\mathrm{mm}$ ). The objective of the optimization is to find the best values of the design parameters to meet the design objectives; i.e., maximizing the bending angle of the finger $(\theta)$ and minimizing the joint dimensions (length and diameter) under an approximate value of applied pressure to the joint $\left(P_{1}\right)$. The amount of this pressure is numerically determined based on the $80 \%$ of the pressure that causes the joint to burst with $1 \mathrm{~mm}$ thickness of the chamber wall $\left(H_{1}\right)$ which is equal to $14 \mathrm{kPa}$. Figure 4 summarizes the design parameters under investigation, including the main geometrical parameters of the joint. The range of variation for each design parameter is tabulated in Table I. The lower and the upper bounds are specified based on the fabrication considerations and also the dimensions of the finger which are expected to be equivalent to the human finger. The pressure

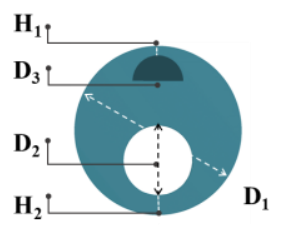

$$
\begin{array}{ll}
D_{1}: \text { Joint diameter } & \\
D_{2}: \text { Hole diameter } & H_{2}: \text { Hole to wall thickness } \\
D_{3}: \text { Chamber diameter } & L_{1}: \text { Joint length } \\
H_{1}: \text { Chamber to wall thickness } & L_{2}: \text { Chamber length }
\end{array}
$$

Figure 4.The geometrical optimization parameters.

TABLE I. RANGES FOR DESIGN OPTIMIZATION PARAMETERS

\begin{tabular}{lcc}
\hline Design Parameters & Lower Bound & Upper Bound \\
\hline Joint length - $L_{1}(\mathrm{~mm})$ & 40 & 60 \\
Chamber length $-L_{2}(\mathrm{~mm})$ & 30 & 50 \\
Joint diameter $-D_{1}(\mathrm{~mm})$ & 20 & 40 \\
Chamber diameter $-D_{3}(\mathrm{~mm})$ & 6 & 10 \\
Chamber to wall thickness $-H_{1}(\mathrm{~mm})$ & 1 & 2 \\
Hole to wall thickness $-H_{2}(\mathrm{~mm})$ & 1 & 5 \\
Pressure inside the link $-P_{2}(\mathrm{kPa})$ & 110 & 150 \\
\hline
\end{tabular}

inside the link $\left(P_{2}\right)$ is taken into account as another design parameter and the range of variation is selected in a way that a sensible variation can be observed in the stiffness of the finger. FEM is employed to solve the relevant equations for flexible materials numerically in the aim to evaluate the candidates within the design search space. ANSYS Workbench with the option of large nonlinear deformation for hyperelastic materials is used as the framework for solving these numerical equations and performing optimizations. As for selecting the materials, two variations of platinum-catalyzed silicones are nominated to fabricate the actuator module: Ecoflex 00-30 and 00-50, the code numbers referring to the material's shore hardness. As studied by Elsayed et al. [17], both the silicones exhibited the same bending behavior; however, lower pressure is required to deform the joint made of Ecoflex 00-30; accordingly, in this work, this material is selected to fabricate the joint. As for the link, Dragonskin 00-30 is selected. This is due to the direct interaction of the link with objects and consequently, the need for higher stiffness. Simulating the behaviors of these materials, silicone rubber is presumed as an isotropic and hyperplastic material. According to [18], for the Ecoflex 00-30, the third-order Ogden model $(N=3)$ for the strain energy potential is expressed with $\mu$ and $\alpha$ as the empirical parameters (1). The parameters values $\mu_{1}=22 \mathrm{kPa}$, $\alpha_{1}=1.3, \mu_{2}=0.4 \mathrm{kPa}, \alpha_{2}=5, \mu_{3}=-2 \mathrm{kPa}$, and $\alpha_{3}=-2$ show the best curve fit with the experimental stress-strain data of the mechanical tests.

$$
U=\sum_{i=1}^{N} \frac{2 \mu_{i}}{\alpha_{i}^{2}}\left(\lambda_{1}^{\alpha_{i}}+\lambda_{2}^{\alpha_{i}}+\lambda_{3}^{\alpha_{i}}-3\right)
$$

As for Dragon skin 00-30, the second order Yeoh model is chosen due to the promising fitting with the stress-strain data of the mechanical tests with the parameter values of $N=2, C_{10}=1.190 \mathrm{kPa}$, and $C_{20}=23.028 \mathrm{kPa}$ [17]. This model can be presented for incompressible materials as in (2).

$$
U=C_{10}\left(\bar{I}_{1}-3\right)+C_{20}\left(\bar{I}_{1}-3\right)^{2}
$$

Due to the large deformations in the joint structure, SOLID187 elements are used to mesh the model. These elements with quadratic displacement behavior are defined by ten nodes having three degrees of freedom at each node. This characteristic along with capabilities such as plasticity, hyperelasticity, creep, stress stiffening, large deflection, and large strain make them well suited to irregular model meshes (such as those produced in this analysis). Fixed support boundary conditions are applied to the beginning of both the link and the joint while the tips are set free to move. As for simulating the pressures in the joint and the link chambers $\left(P_{1}\right.$ $\left.\& \quad P_{2}\right)$, constant normal pressure boundary conditions are considered with relevant values. Figure 5a illustrates the results of the bending simulation of a sample up to 90 degrees under the actuation pressure of $14 \mathrm{kPa}$.

\section{Sensitivity Analysis}

Before optimization, local sensitivity analysis helps to find the positive or negative effect of each design parameter on the objective output. This analysis is useful when a large number 

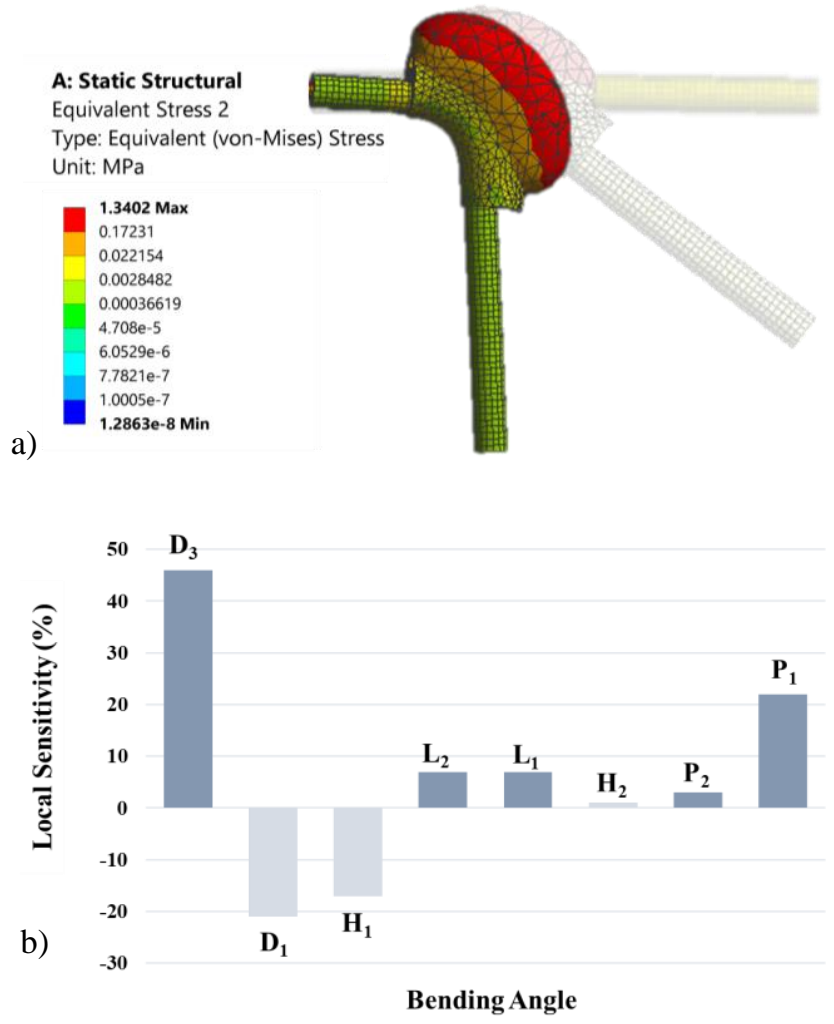

Figure 5. a) FEA simulation of a finger up to 90 degrees under the actuation pressure of $14 \mathrm{kPa}$. b) local sensitivity of the optimized result to each design parameter.

of variables exist and need to figure out the most critical design parameters to reduce the computational cost of the optimization [19]. The local sensitivity is calculated according to the (3),

$$
\text { Local Sensivity }_{(\%)}= \pm \frac{\left(\text { output }_{\max }-\text { output }_{\min }\right)_{\text {local }}}{\left(\text { output }_{\max }-\text { output }_{\min }\right)_{\text {global }}} \times 100
$$

Where (output $t_{\max }-$ output $\left._{\min }\right)_{\text {local }}$ is calculated when one input value varies and other values are assumed to be constant in particular geometry and (output $t_{\max }-$ output $\left._{\min }\right)_{\text {global }}$ is calculated when all the inputs vary. Figure 5 b presents the local sensitivity analysis of each design parameter in percent, as it can be observed, the chamber diameter $\left(D_{3}\right)$ and the actuation pressure $\left(P_{1}\right)$ are estimated to be the most important elements with direct relation to the bending angle; in other words, compared to other variables, increasing these two elements results in more intense positive changes in the final bending angle. The joint diameter $\left(D_{1}\right)$ and the chamber to wall distance $\left(H_{1}\right)$ stand in the next ranks of the most influential parameters, but in reverse relation with the main objective which means that the reduction in the values of these parameters causes the final bending angle to increase. On the other hand, the variations of the hole to wall distance $\left(\mathrm{H}_{2}\right)$ and the pressure inside the link $\left(P_{2}\right)$ are estimated to be almost ineffective to the main objectives of the optimization and hence to be neglected; nevertheless, it should be noted that $P_{2}$ is an important parameter for changing the finger stiffness and thus the applied fingertip force. The effect of this parameter will be discussed in the following of this paper. As for $\mathrm{H}_{2}$, the value is determined according to manufacturing considerations and is set to $2 \mathrm{~mm}$. The low value of this parameter would result in aligning the surfaces of the joint and the link in the bending direction. This eventually leads to a uniform smooth surface in that area which can be beneficial in future possible grasping applications.

\section{Optimization Process}

After identifying the influential design parameters with local sensitivity analysis, an optimization analysis must be conducted. The goal is to maximize the bending angle up to 90 degrees and simultaneously minimizing the length and diameter of the joint (miniaturizing the dimension of the finger to be more like a human finger) under the applied pressure to the joint $\left(P_{1}\right)$ around $14 \mathrm{kPa}$. The optimization process and perquisites are shown in the flowchart in Figure 6. Due to the multiple numbers of design parameters, objectives and constraints, the Adaptive Multiple-Objective optimization method is selected to find the global optimum parameters. This method is a type of the Non-dominated Sorted Genetic Algorithm (NSGA-II) which is based on controlled elitism concepts [16]. This average value is selected through trial and error so that the finger with the specified ranges of geometrical dimensions can bend up to 90 degrees and not burst. The calculation converged by generating 400 samples with 50 samples per iteration and finding the best candidate in 8 iterations. Furthermore, during the process, if the FEM simulation of a sample fails (e.g. bursting), it will be

\section{START}

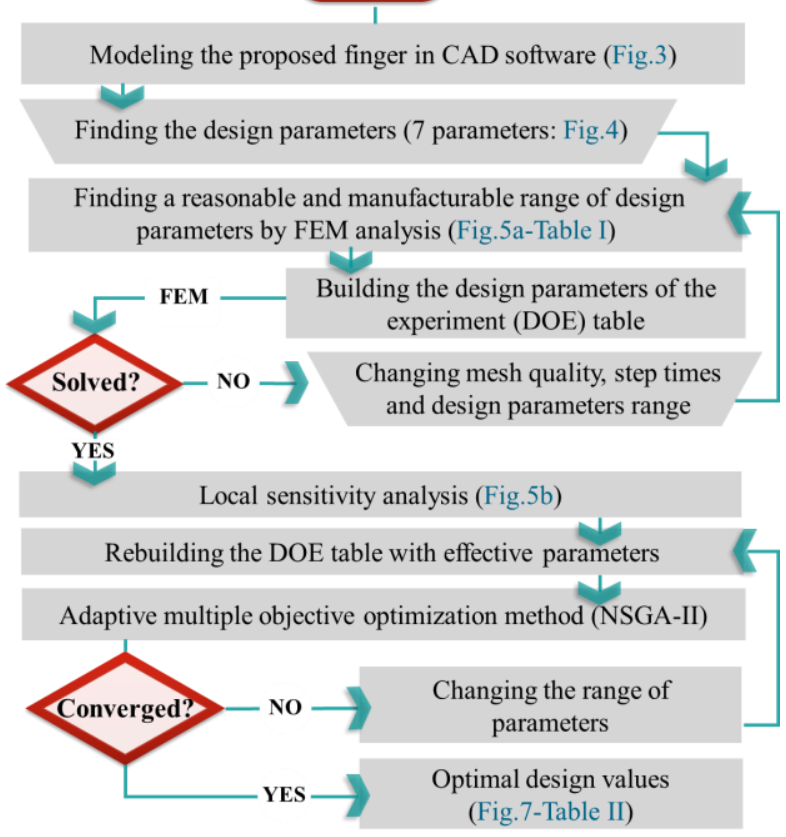

Figure 6. Flowchart of the proposed optimization methodology. The trapezoid shapes represent a manual operation and the other rectangular shapes are the automated process. 
TABLE II. FEM OPTIMIZED PARAMETERS

\begin{tabular}{lc}
\hline Design Parameters & Optimized value \\
\hline Joint length - $L_{1}(\mathrm{~mm})$ & 44.6 \\
Chamber length $-L_{2}(\mathrm{~mm})$ & 42.5 \\
Joint diameter - $D_{1}(\mathrm{~mm})$ & 22.3 \\
Chamber diameter $-D_{3}(\mathrm{~mm})$ & 9.14 \\
Chamber to wall thickness $-H_{1}(\mathrm{~mm})$ & 1.68 \\
\hline
\end{tabular}

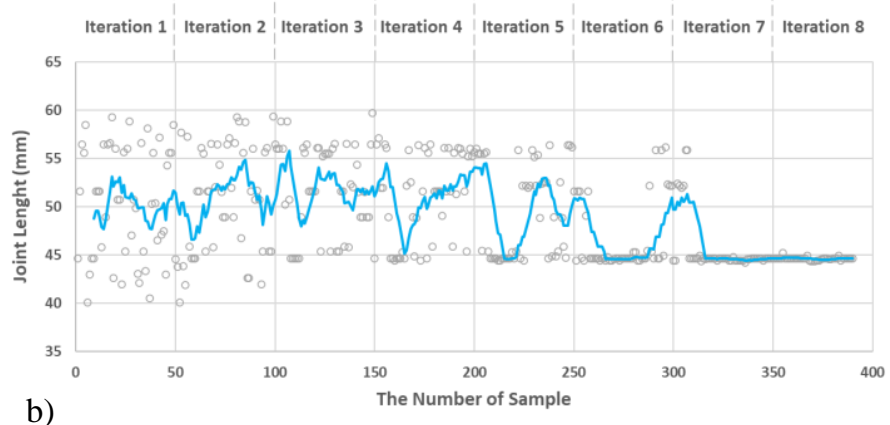

b)

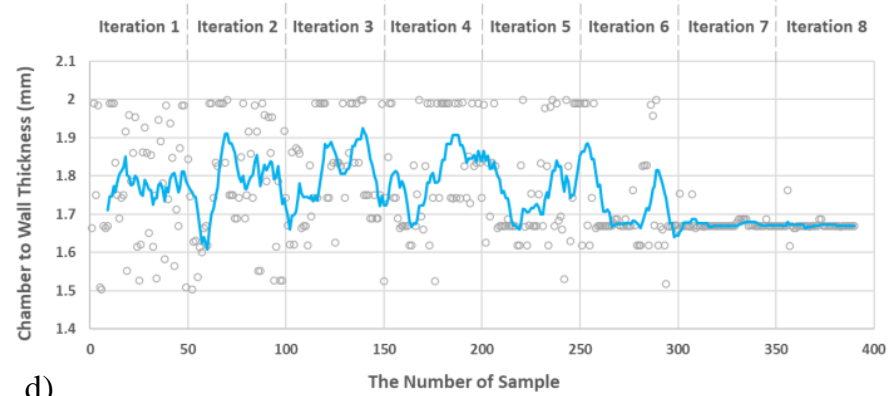

d)

The Number of Sample

Figure 7. a-e) Optimization of the proposed soft finger: convergence of the design parameters to the final optimized values (blue lines show the moving average of each design parameter).

eliminated and replaced by a new sample. Totally, 146 new samples have been generated and replaced the failed ones. The optimization charts are shown in Figures 7a-7e. In these figures, empty circles show the samples and the blue lines illustrate the moving average which is the best-fitted line that represents the convergence trend of the samples to the optimized values. Table II summarizes the eventual optimized values. These dimensions will be used to manufacture the prototype of the finger. The molds are printed with the Ultimaker3 3D printer. Thanks to recent advances in 3D printing technology, the fabrication process of soft components has been facilitated significantly which leads to producing more complex parts with higher precision. For each silicone, two liquid parts should be mixed with the same ratio followed by 2-3 minutes vacuum degassing to eliminate any entrapped air bubbles. This will be done by placing the molds in a vacuum chamber.
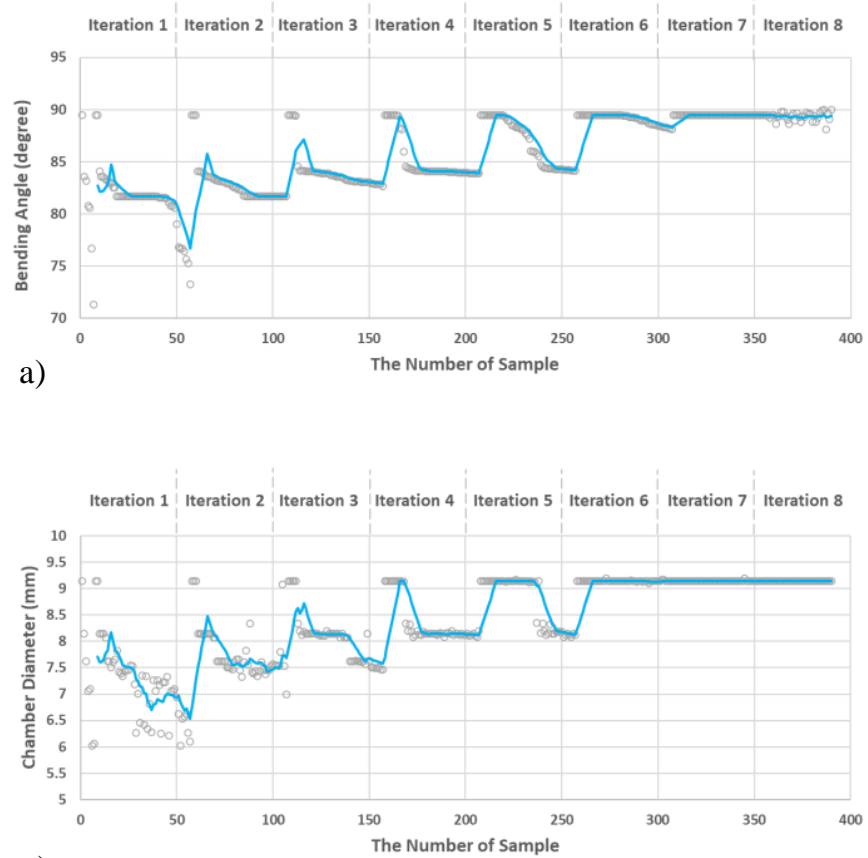

c)

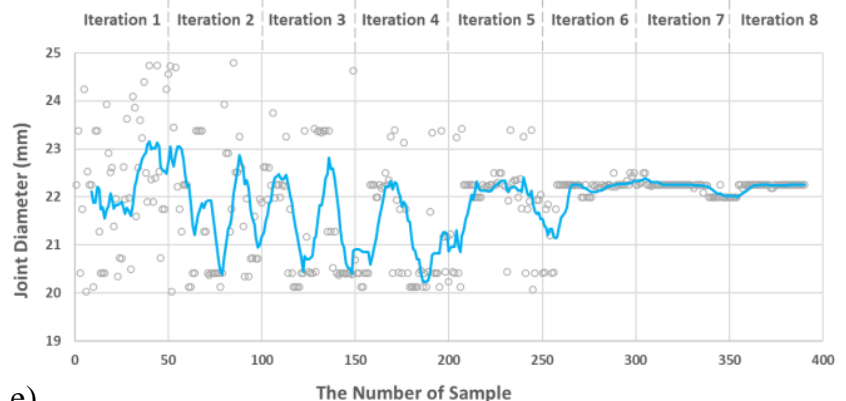

e)

\section{Results and Discussions}

Towards a better understanding of the finger mechanism, the workspace analysis is evaluated (Figure 8). The kinematic model of the finger is considered as one joint and two links. The first link (red) is fixed and the second one (blue) can bend up to 90 degrees in the XY plane. The whole finger can rotate around the $\mathrm{X}$-axis about 300 degrees. By changing the position of the joint, the bending point and consequently lengths of the two links are changed. From an earlier section, the length of the finger and joint were presumed as the range of 15 and 4.46 $\mathrm{cm}$. The bending point (center of joint) can be moved along the $\mathrm{X}$-axis from 2.5 to $12.5 \mathrm{~cm}$, the resulting workspace of every point touched by a fingertip in 2D (Figure 8a) and 3D (Figure 8b) is calculated. The 2D workspace comparison between the proposed finger and a traditional design shows that changing the position of bending increases the number of

\section{Workspace Analysis}



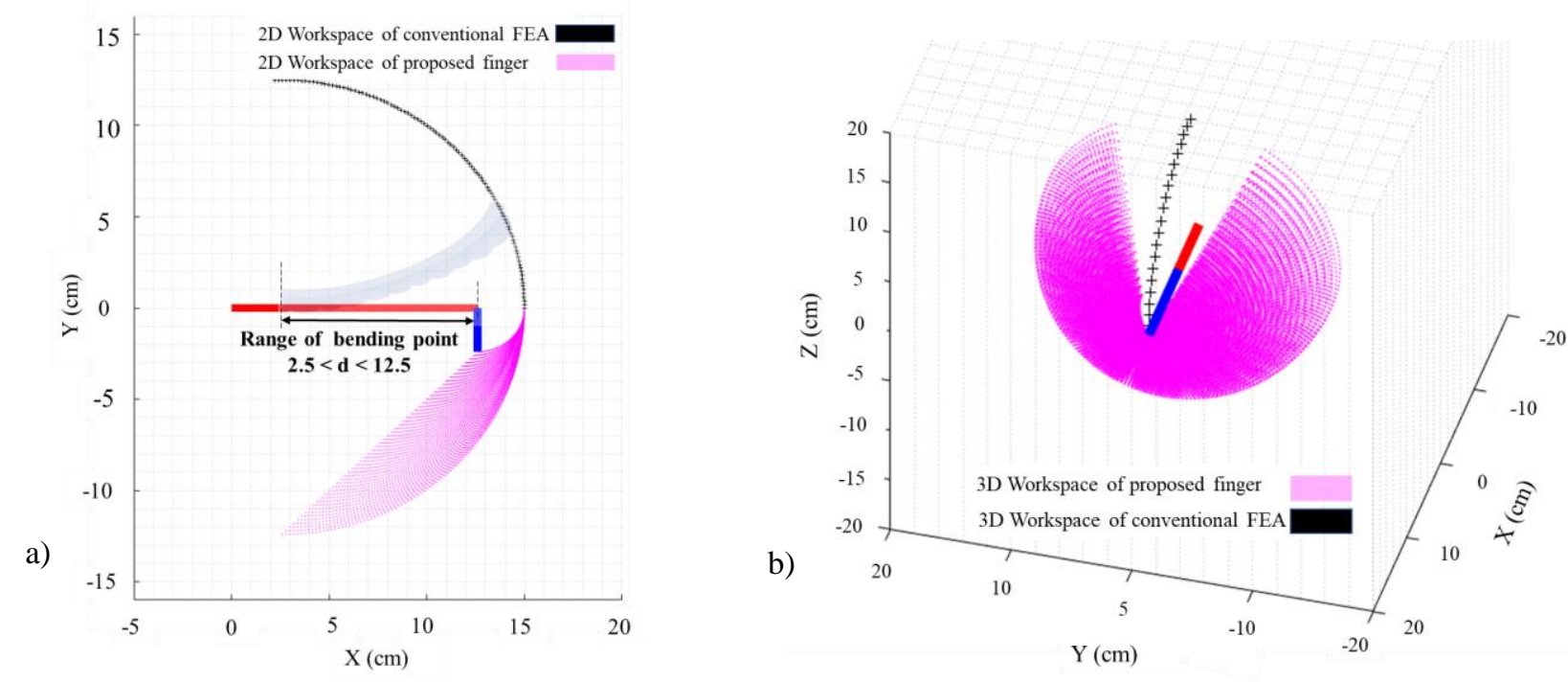

Figure 8. Workspace evaluation of proposed finger compared to conventional FEAs with $12.5 \mathrm{~cm}$ length a) in 2D space. b) in 3D space (the finger can rotate around its axis of about $300^{\circ}$ ).

accessible points while the tipping point workspace of previous traditional FEAs is assumed to be a constant arc [20].

\section{Experimental Results}

Validating the numerical model introduced in the previous sections, the fabricated finger undergoes two sets of experiments, i.e., bending and force tests. Figure 9a shows the prototype assembled to conduct the experimental tests.

a)
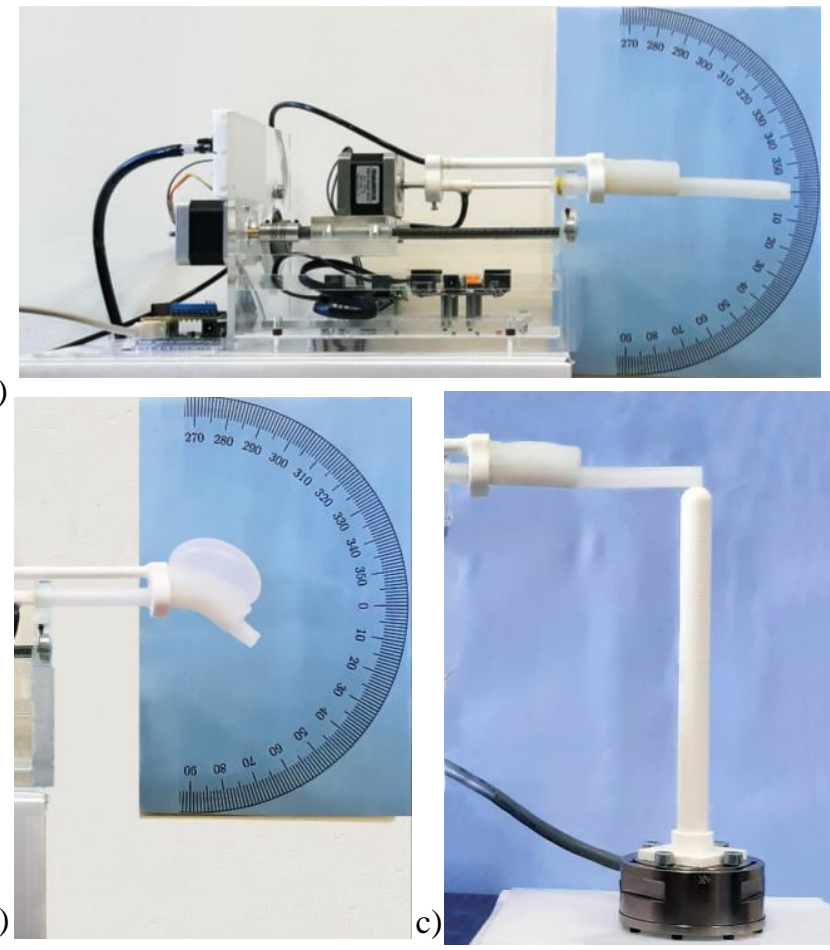

Figure 9. a) The overall view of the assembled prototype. b) test bench for measuring the joint angle. c) test bench for measuring the fingertip force.
Controlling the whole process including reading sensors, switches, and electric motors are performed by an Arduino Uno board which is connected to the computer via a USB wire. A $12 \mathrm{~V} 350 \mathrm{kPa}$ air pump is used for supplying the pressurized air for the system. Regulating the pressures $P_{1}$ and $P_{2}$ independently, one solenoid valve and one silicon piezoresistive pressure sensor are embedded in each air stream. The feedback signals transfer from each pressure sensor to the Arduino are used to switch on and off the air pump and the relevant solenoid valve. Two test benches are developed to characterize the bending angle as well as the blocking force of the fingertip. The bending angle of the finger is checked using a printed protractor placed at the joint's center of bending (Figure 9b). As for measuring the force applied by the fingertip, a force sensor is situated below the tipping point of the finger and directly transfers the force data to the computer (Figure 9c). Due to the weight of the link, at the initial state, a deflection of 10 degrees at the tipping point of the finger can be observed which will be resolved by applying the pressure $P_{2}$ inside the link. Different pressures $P_{1}$ are applied to the joint and the consequent bending angles are measured. These angles are compared with the numerical results in Figure 10a. It can be noticed that there is an acceptable agreement between the experimental and numerical data which can be taken as the validity of the model and thus the optimization results. As the second test with the assembled prototype, the force at the tipping point of the finger is measured as a function of different parameters including the longitudinal position of the joint and the stiffening pressure ( $P_{2}$ ). All the tests are conducted under the actuation pressure of $P_{1}=14 \mathrm{kPa}$. The results are presented in Figure 10b. As illustrated, by changing the position of the joint toward the tip of the finger, the applied force increases by almost three times. Furthermore, applying the pressurized air into the link results in higher stiffness and thus a higher amount of force up to 650 $\mathrm{mN}$ which is twice the initial value. 

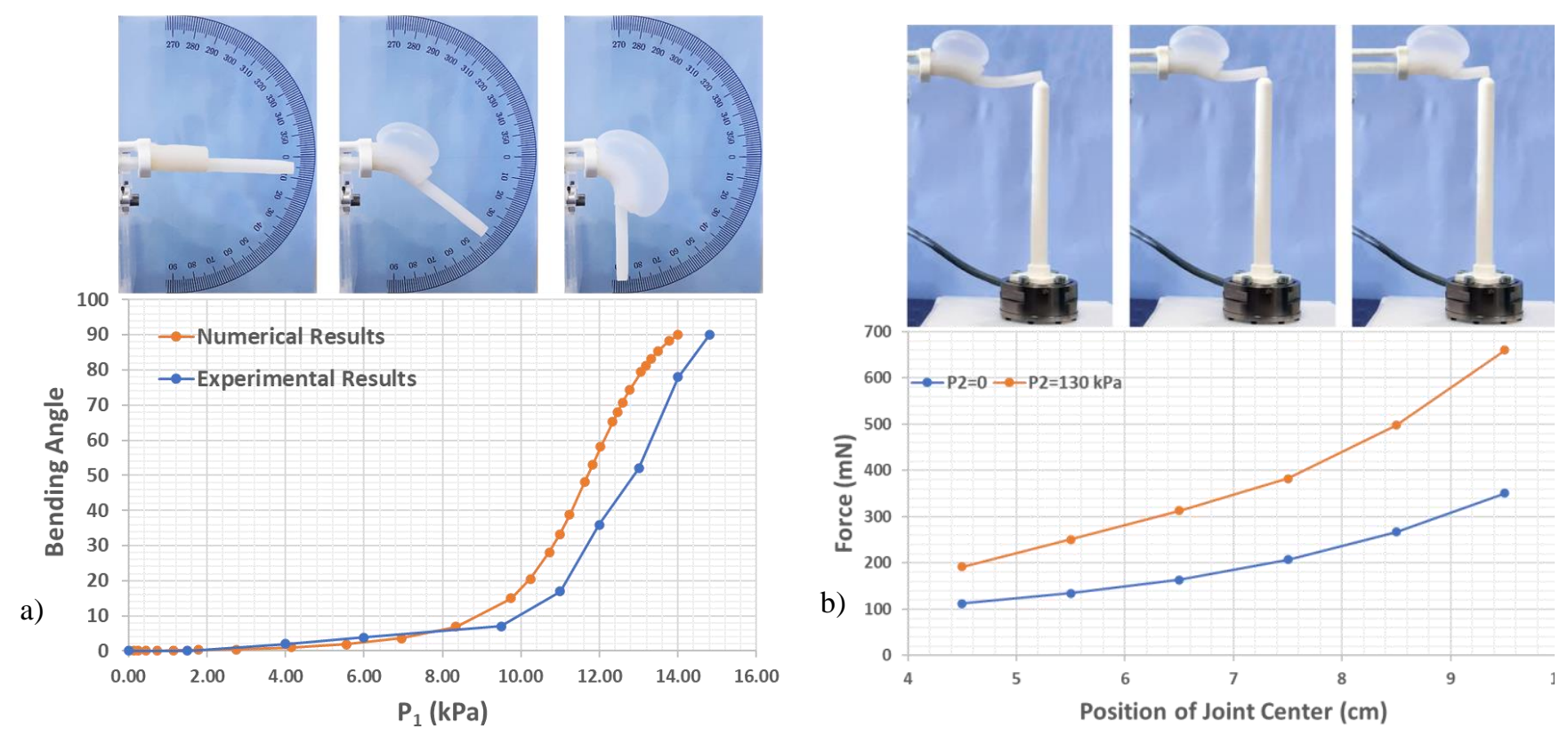

Figure 10. a) Bending angles of the finger under different applied pressures, comparison between ANSYS FEM numerical simulation and experimental results b) fingertip force test results as a function of stiffening pressure $\left(P_{2}\right)$ and joint longitudinal position.

\section{Conclusions}

In this paper, an innovative variable stiffness soft finger with a fluid-actuated movable joint was introduced and optimized in terms of its main characteristics. The finger mainly consists of one soft sliding and rotating joint as the bending actuator and a soft link as the body. Applying pressurized air into the joint's chamber, the joint and consequently, the link bends in a specific direction. The location and the direction of the bending can be changed by sliding the joint longitudinally along the link and rotating it around its main axis using two electric motors. The variable length of the finger with the capability of bending in different directions results in a large diversity of configurations. The workspace analysis exhibited the advantage of this reconfigurability by extending the available workspace of the fingertip in contrast to conventional FEAs. Local sensitivity of the design parameters involved in the problem was analyzed. Optimization over the important parameters was performed to minimize the joint dimensions and maximize the bending angle of the finger. The model included a large number of design parameters with nonlinear relations which made the prediction of the deformation difficult, i.e., small changes in each one can lead to large deviations in the final results. Hence, implementing the optimizing process is necessary to investigate the acceptable and manufacturable range of these parameters. The optimal geometrical parameters were used for fabricating a prototype that validates the numerical model. Another experiment was designed to study the amount of force applied by the fingertip. It was shown that the longitudinal location of the joint and also the pressure inside the link $\left(P_{2}\right)$ were highly effective to this force. The wide range of force applied to the fingertip as well as the diversity of possible configurations to reach a given target leads to a variety of strategies to deal with situations such as the variable amount of force required or presence of any obstacles in the workspace. Besides, optimizing the dimension of the finger allowed us to reduce the volume of the required air and consequently the response time. The experiments showed that the joint can bend up to 90 degrees in less than three seconds, in contrast with previous approaches (i.e. SMPs or LMPAs).

\section{Future Works}

As for the future works, the idea of using these fingers in a dexterous gripper with miniaturized pumps and electric motors will be studied. Furthermore, combining sensitivity analysis and NSGA-II optimization provides a design basis for this future gripper, especially by considering an energy criterion.

\section{Acknowledgment}

This work has been sponsored by the French government research program "Investissements d'Avenir" through the IDEX-ISITE initiative 16-IDEX-0001 (CAP20-25).

\section{References}

[1] D. Trivedi, C. D. Rahn, W. M. Kier, and I. D. Walker, "Soft robotics: Biological inspiration, state of the art, and future research," Applied bionics and biomechanics, vol. 5, no. 3, pp. 99-117, 2008.

[2] A. D. Marchese and D. Rus, "Design, kinematics, and control of a soft spatial fluidic elastomer manipulator," The International Journal of Robotics Research, vol. 35, no. 7, pp. 840-869, 2016. 
[3] J. Shintake, V. Cacucciolo, D. Floreano, and H. Shea, "Soft Robotic Grippers," Advanced Materials, p. 1707035, 2018.

[4] R. F. Shepherd et al., "Multigait soft robot," Proceedings of the national academy of sciences, vol. 108, no. 51, pp. 20400-20403, 2011.

[5] W. McMahan et al., "Field trials and testing of the OctArm continuum manipulator," in Proceedings 2006 IEEE International Conference on Robotics and Automation, 2006. ICRA 2006., 2006, pp. 2336-2341.

[6] M. Cianchetti, T. Ranzani, G. Gerboni, I. De Falco, C. Laschi, and A. Menciassi, "STIFF-FLOP surgical manipulator: mechanical design and experimental characterization of the single module," in $20103 \mathrm{rd}$ IEEE RAS and EMBS International Conference, 2010, pp. 461-466.

[7] P. Polygerinos, Z. Wang, K. C. Galloway, R. J. Wood, and C. J. Walsh, "Soft robotic glove for combined assistance and at-home rehabilitation," Robotics and Autonomous Systems, vol. 73, pp. 135-143, 2015.

[8] H. K. Yap, H. Y. Ng, and C.-H. Yeow, "High-force soft printable pneumatics for soft robotic applications," Soft Robotics, vol. 3, no. 3, pp. 144-158, 2016.

[9] Y. Yang, Y. Chen, Y. Li, M. Z. Chen, and Y. Wei, "Bioinspired robotic fingers based on pneumatic actuator and 3D printing of smart material," Soft robotics, vol. 4, no. 2, pp. 147-162, 2017.

[10] Y. Yang, Y. Chen, Y. Li, Z. Wang, and Y. Li, "Novel variable-stiffness robotic fingers with built-in position feedback," Soft robotics, vol. 4, no. 4, pp. 338-352, 2017.

[11] Y. Hao et al., "A eutectic-alloy-infused soft actuator with sensing, tunable degrees of freedom, and stiffness properties," Journal of Micromechanics and Microengineering, vol. 28, no. 2, p. 024004, 2018.

[12] H. K. Yap, J. H. Lim, F. Nasrallah, J. C. Goh, and R. C. Yeow, "A soft exoskeleton for hand assistive and rehabilitation application using pneumatic actuators with variable stiffness," in 2015 IEEE international conference on robotics and automation (ICRA), 2015, pp. 4967-4972.
[13] B. E. Schubert and D. Floreano, "Variable stiffness material based on rigid low-melting-point-alloy microstructures embedded in soft poly (dimethylsiloxane)(PDMS)," Rsc Advances, vol. 3, no. 46, pp. 24671-24679, 2013.

[14] Y. Elsayed, C. Lekakou, T. Geng, and C. M. Saaj, "Design optimisation of soft silicone pneumatic actuators using finite element analysis," in 2014 IEEE/ASME International Conference on Advanced Intelligent Mechatronics, 2014, pp. 44-49.

[15] G. Decroly, B. Mertens, P. Lambert, and A. Delchambre, "Design, characterization and optimization of a soft fluidic actuator for minimally invasive surgery," International Journal of Computer Assisted Radiology and Surgery, vol. 15, no. 2, pp. 333-340, 2020.

[16] K. Deb, A. Pratap, S. Agarwal, and T. Meyarivan, "A fast and elitist multiobjective genetic algorithm: NSGA-II," IEEE transactions on evolutionary computation, vol. 6, no. 2, pp. 182-197, 2002.

[17] Y. Elsayed et al., "Finite element analysis and design optimization of a pneumatically actuating silicone module for robotic surgery applications," Soft Robotics, vol. 1, no. 4, pp. 255-262, 2014.

[18] D. Steck, J. Qu, S. B. Kordmahale, D. Tscharnuter, A. Muliana, and J. Kameoka, "Mechanical responses of Ecoflex silicone rubber: compressible and incompressible behaviors," Journal of Applied Polymer Science, vol. 136, no. 5, p. 47025, 2019.

[19] A. F. Release, "16.2, ANSYS ${ }^{\circledR}$ Academic Research, Help System," Fluent Theory and User's Guide, 2016.

[20] P. Polygerinos et al., "Modeling of soft fiber-reinforced bending actuators," IEEE Transactions on Robotics, vol. 31, no. 3, pp. 778-789, 2015.

The authors are with Université Clermont Auvergne, CNRS, SIGMA Clermont, Institut Pascal, F-63000 ClermontFerrand, France. Emails: \{amir. pagoli, frederic.chapelle, juan-antonio.corrales-ramon, youcef.mezouar, yuri.lapusta\} @ sigma-clermont.fr 